\title{
Patellofemoral pain in athletes
}

This article was published in the following Dove Press journal:

Open Access Journal of Sports Medicine

12 June 2017

Number of times this article has been viewed

\author{
Wolf Petersen' \\ Ingo Rembitzki \\ Christian Liebau ${ }^{3}$ \\ 'Department of Orthopaedic and \\ Trauma Surgery, Martin Luther \\ Hospital, Grunewald, Berlin; \\ ${ }^{2}$ German Sport University Cologne, \\ ${ }^{3}$ Asklepios Clinic, Bad Harzburg, \\ Germany
}

Correspondence: Wolf Petersen

Klinik für Orthopädie und Unfallchirurgie, Martin Luther Krankenhaus, Caspar

Theyss Strasse 27-3I, Grunewald, Berlin 14193, Germany

Email w.petersen.berlin@gmail.com
Abstract: Patellofemoral pain (PFP) is a frequent cause of anterior knee pain in athletes, which affects patients with and without structural patellofemoral joint (PFJ) damage. Most younger patients do not have any structural changes to the PFJ, such as an increased Q angle and a cartilage damage. This clinical entity is known as patellofemoral pain syndrome (PFPS). Older patients usually present with signs of patellofemoral osteoarthritis (PFOA). A key factor in PFPS development is dynamic valgus of the lower extremity, which leads to lateral patellar maltracking. Causes of dynamic valgus include weak hip muscles and rearfoot eversion with pes pronatus valgus. These factors can also be observed in patients with PFOA. The available evidence suggests that patients with PFP are best managed with a tailored, multimodal, nonoperative treatment program that includes short-term pain relief with nonsteroidal anti-inflammatory drugs (NSAIDs), passive correction of patellar maltracking with medially directed tape or braces, correction of the dynamic valgus with exercise programs that target the muscles of the lower extremity, hip, and trunk, and the use of foot orthoses in patients with additional foot abnormalities.

Keywords: anterior knee pain, dynamic valgus, hip strength, rearfoot eversion, single leg squat, hip strength

\section{Introduction}

Patellofemoral pain (PFP) is the accepted term for localized pain of the anterior aspect of the knee..$^{1,2}$ The prevalence of PFP is high; ${ }^{1,2}$ it affects $11-17 \%$ of patients who present to general practitioners. ${ }^{1,2}$ PFP is typically associated with activities that load the patella, such as ascending or descending stairs, jumping, running, and squatting. ${ }^{1-4}$ Other symptoms often associated with PFP are crepitus and mild/moderate joint effusion. ${ }^{1}$

It was recently reported that $\sim 25 \%$ of recreational athletes diagnosed with PFP will stop participating in sports because of knee pain. ${ }^{5}$ PFP frequently affects running and jumping athletes. ${ }^{6}$ In an observational study of 810 adolescent basketball players, the overall prevalence of PFP was $25 \%$, with $\sim 26 \%$ of female and $18 \%$ of male players affected. ${ }^{6}$ Functional patellofemoral pain syndrome (PFPS), a synonym for nonspecific PFP, ${ }^{7}$ was the most common diagnosis with an overall prevalence of $6.4 \% .{ }^{6}$ Other less common diagnoses included Sinding-Larsen-Johansson disease (4.8\%), Osgood-Schlatter disease (2.5\%), and plica syndrome (2.3\%). ${ }^{6}$ These observations show that PFP can affect patients with and without any structural damage to the patellofemoral joint (PFJ). ${ }^{7}$ Structural damage that is sometimes associated with PFP includes chondral and osteochondral damage, osteoarthritis (OA), overuse injuries of the extensor apparatus (tendonitis and insertional tendinosis), and patellar 
instability. ${ }^{9}$ It has been recommended that patients with a history of dislocations or subluxations should be considered separately from other patients with PFP because this subgroup of patients frequently have biomechanical risk factors that require different treatment approaches. ${ }^{1}$ The same is true for those with overuse injuries of the extensor apparatus or cartilage damage.

Nonspecific PFP is a common cause of "anterior knee pain" and mainly affects young athletes without any structural abnormalities. ${ }^{7}$ Patellofemoral osteoarthritis (PFOA) predominantly affects older athletes with cartilage damage and bone marrow lesions. ${ }^{7}$ Well-known causes of PFOA are trauma, deformity, and instability (Figure 1). PFP and PFOA are two separate clinical entities. However, a recent systematic review suggested that nonspecific PFP in young patients could be a precursor to subsequent PFOA. ${ }^{8}$

The purpose of this article is to summarize the evidence regarding the pathophysiology of nonspecific PFP and PFOA and to review the treatment of athletes with these conditions.

\section{PFP pathophysiology or pathomechanics \\ Patella maltracking and PFP}

A recent study using $3 \mathrm{~T}$ magnetic resonance imaging (MRI) demonstrated that structural abnormalities of the patellofemoral cartilage are not associated with PFP. ${ }^{9}$ In this study, minor patellar cartilage defects or patellar bone marrow lesions were frequently seen in both PFP patients and subjects without PFP. These observations are in accordance with the findings of arthroscopic studies. ${ }^{9}$

A recent systematic review examined the association of other PFJ imaging features, such as bisect offset, congruence angle, and patellar tilt, with PFP. ${ }^{10}$ In this study, an increased MRI bisect offset, increased patellar tilt, and increased congruence angle under load and without load were associated with PFP. ${ }^{10}$ All of these imaging parameters are indices of patellar maltracking. ${ }^{7}$ This provides strong scientific evidence

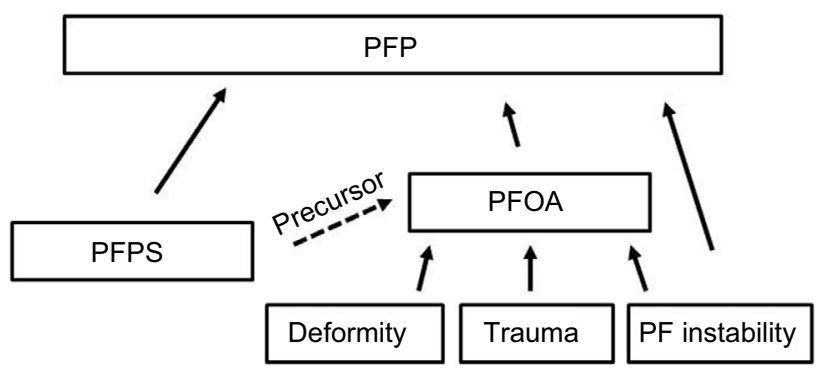

Figure I Schematic drawing showing the relationship of PFP, PFPS, and PFOA. Abbreviation: PFP, patellofemoral pain; PFPS, patellofemoral pain syndrome; PFOA, patellofemoral osteoarthritis; PF, patellofemoral. that PFP is associated with patellar maltracking. ${ }^{10}$ Interestingly, both patellar tilt and bisect offset were increased when MRI was performed under load..$^{10}$ Wilson et $\mathrm{al}^{11}$ used skin markers and an optoelectronic motion capture system to examine patellar glide in young patients with PFPS while in a standing position and while squatting. They found that the patellae of patients with PFPS had significantly increased lateral translation (maltracking), lateral patellar spin, and a tendency toward increased lateral tilt compared with the patellae of healthy subjects. ${ }^{11}$ The observation that patellar maltracking is most severe in the upright or weight-bearing (squat) positions indicates that muscular activity is the primary determinant of patellar position in PFP patients.

Other studies have also investigated the association between patellar maltracking and PFP. The Health ABC study examined the relationship between patellar tracking and PFOA in a cohort of 3075 men and women aged between 70 and 79 years. ${ }^{12}$ In this population, patellar maltracking was associated with PFOA progression. ${ }^{12}$ Lateral displacement and patellar tilt predisposed to lateral PFOA progression, while medial displacement was predictive of medial PFOA progression. ${ }^{12}$ Greater patellar tilt was associated with more severe pain. ${ }^{12}$ In the Multicenter Osteoarthritis (MOST) and Framingham Osteoarthritis (FOA) studies, PFP was associated with lateral patellofemoral cartilage damage and concomitant bone marrow lesions but not with medial cartilage damage. ${ }^{13}$ Similarly, a recent review concluded that there is strong evidence that PFOA is also associated with signs of patellofemoral instability, such as trochlear dysplasia and frontal plane knee alignment. ${ }^{1}$

In conclusion, patellar maltracking plays a key role in patients with PFP related to PFPS or PFOA (Figure 2).

\section{Dynamic valgus}

It is well known that the direction of the force generated by the quadriceps influences patellar tracking because the quadriceps force vector is lateral to the center of the joint line. ${ }^{7,14-16}$ The quadriceps angle ( $Q$ angle) is a static measure of the quadriceps force vector. ${ }^{7,14}$ The $\mathrm{Q}$ angle is formed by a line from the anterior superior iliac spine to the midpoint of the patella and a line from the midpoint of the patella to the tibial tubercle (Figure 3). ${ }^{14}$ The normal value for this angle is $13.5 \pm 4.5^{\circ}$ (in healthy subjects between 18 and 35 years old). ${ }^{14,16}$ An increase in the $Q$ angle increases the lateral force on the patella. ${ }^{7,14,16}$ It has been shown that larger Q angles are associated with femoropatellar instability and with PFOA. ${ }^{14,15}$ However, the use of the $\mathrm{Q}$ angle to predict PFP is controversial. A recent systematic review reported that greater $\mathrm{Q}$ 
A

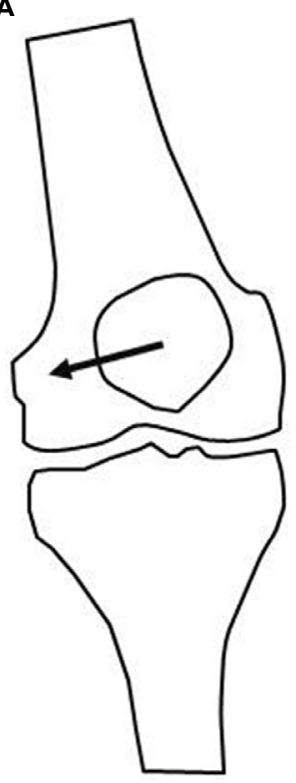

Figure 2 Drawing showing patellar maltracking in PFP patients.

Notes: (A) Patients with PFPS have increased lateral shift (arrow). (B) Inpatients with PFOA, lateral maltracking promotes disease progression.

Abbreviations: PFPS, patellofemoral pain syndrome; PFOA, patellofemoral osteoarthritis.

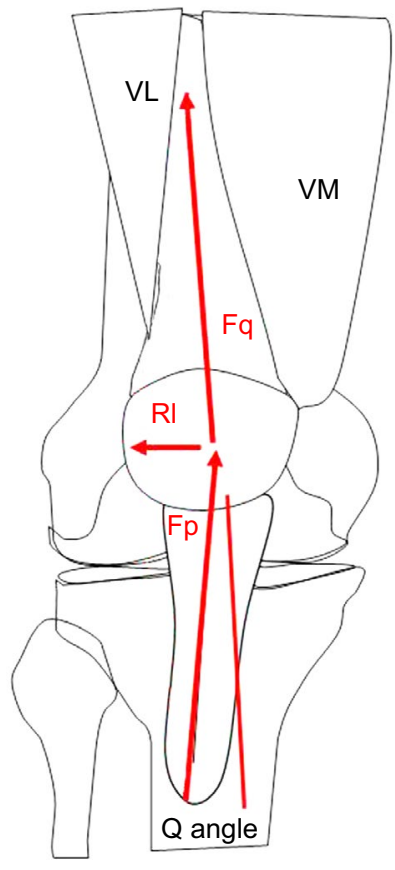

Figure 3 Schematic drawing showing the $Q$ angle.

Abbreviations: VM, vastus medialis muscle; VL, vastus lateralis muscle; Fq, quadriceps force; Fp, patellar tendon force; R, resultant force.

angles were associated with PFP, ${ }^{17}$ but other studies have not found correlations between Q angle and PFP in young patients. ${ }^{18-21}$ This suggests that there may be a subgroup of patients in whom PFP is not the result of a structural fault. ${ }^{7}$ This hypothesis is supported by Dierks et $\mathrm{al}^{22}$ who showed that among runners with PFP, there was a subgroup of patients with functional knee valgus. The search for an explanation for this seeming contradiction (patellar maltracking without increased $Q$ angle) led to the concept of a dynamic, or functional, valgus malalignment.?

Myer et $\mathrm{a}^{23}$ examined dynamic lower limb alignment in female middle- and high-school basketball players and found that the athletes who developed new PFP had increased knee abduction moments of the symptomatic limb. Nakagawa et $\mathrm{a}^{24}$ found increased hip adduction and knee abduction in patients with PFPS. Souza and Powers ${ }^{25}$ showed that females with PFPS demonstrated greater peak hip internal rotation compared with controls. These results highlight the existence of a subgroup of patients with PFP who do not have a structural valgus deformity but instead have a dynamic valgus malalignment caused by internal rotation of the femur and tibia (Figure 4). ${ }^{7}$ Several studies have shown that dynamic valgus malalignment is more frequently observed in female athletes than in male athletes; ${ }^{6,26,27}$ this may explain the higher prevalence of PFP in young female athletes. ${ }^{28}$

Dynamic valgus malalignment influences patellar tracking because the lateralized quadriceps force vector subsequently leads to lateralization of the patella. ${ }^{29}$ Souza et a ${ }^{29}$ measured femoral and patellar rotation in PFPS patients using open MRI and found that lateral rotation of the patella in females with PFPS was associated with excessive internal rotation,

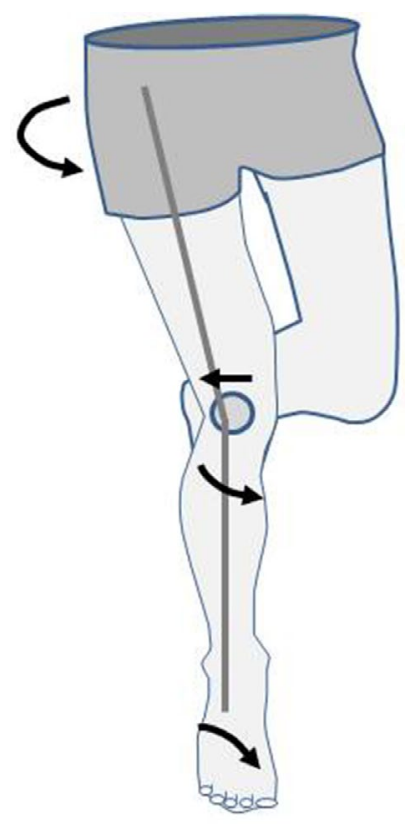

Figure 4 Drawing showing the dynamic valgus.

Notes: This disorder may arise proximally by internal rotation of the femur or distally by internal rotation of the tibia, or both. Internal rotation of the femur might be the result of weakness of the hip muscles. Foot abnormalities might be cause for internal rotation of the tibia (rearfoot eversion or pes pronatus). The valgus malalignement may lead to lateral patella maltracking. 
not torsion, of the femur. One-legged squats are a clinical test used to visualize dynamic valgus. ${ }^{30}$ The one-legged squat task is a qualitative clinical assessment tool that is capable of identifying people with poor hip muscle function. ${ }^{30}$ For this clinical test, participants are instructed to fold their arms across their chest and to squat down as far as possible five times consecutively, in a slow, controlled manner. ${ }^{30}$ Based on five functional criteria (balance, trunk posture, pelvis posture, hip adduction, and knee valgus), the performance on the single leg squat is rated as good, fair, or poor. ${ }^{30}$ Crossley et $\mathrm{al}^{30}$ showed that the good performers exhibited greater hip abduction torque. Therefore, the one-legged squat can be clinically used to evaluate hip muscle function in patients with PFP.

\section{Muscular dysfunction in PFP patients}

There is strong evidence that muscular imbalances play a key role in the pathogenesis of PFP. ${ }^{1}$ Many previous studies have focused on dysfunction of vastus medialis muscle and vastus lateralis muscle in PFP patients because these muscles attach directly to the patella. ${ }^{31-33}$ These studies have shown that an imbalance in the activation of $\mathrm{m}$. vastus medialis obliquus and $\mathrm{m}$. vastus lateralis correlates with lateral patellar tracking in PFP patients. ${ }^{31-33}$

Quadriceps dysfunction is also an important factor in PFOA. ${ }^{1}$ Several studies have shown that quadriceps muscle size, strength, and force are impaired in patients with POA, ${ }^{34-37}$ and quadriceps weakness is considered a risk factor for PFOA. ${ }^{1,38}$ However, despite these results, it remains unclear whether quadriceps dysfunction is the primary cause of patellar maltracking. ${ }^{7}$

There is some evidence in the literature for the involvement of additional muscular imbalances in PFP development. ${ }^{7}$ Several studies have focused on hip muscle dysfunction because of the external rotators of the hip control femoral rotation. ${ }^{39-42}$ These studies have shown that dynamic valgus malalignment does not arise in the knee joint but rather through internal rotation of the femur owing to weakness of hip external rotators and abductors (m. gluteus medius and minimus). ${ }^{39-42} \mathrm{~A}$ systematic review demonstrated strong evidence that females with PFP have decreased hip abduction, external rotation, and extension strength compared with healthy controls. ${ }^{42}$ Padua et $\mathrm{al}^{43}$ showed that decreased strength of the hip abductors and external rotators is related to knee valgus after landing a drop jump. These results show that hip muscle dysfunction is probably an important factor in the pathogenesis of functional valgus malalignment in young patients with PFPS.

Some authors have also detected hip muscle dysfunction in patients with PFOA. ${ }^{44,45}$ Studies have demonstrated lower gluteus minimus and medius peak muscle forces and lower hip abductor strength compared with that of healthy controls. ${ }^{44,45}$ Other muscular abnormalities detected in PFP patients affect the iliotibial tract (m. tensor fascia latae) and the hamstrings. ${ }^{7}$ The influence of the iliotibial tract on patellar tracking ${ }^{46}$ may be anatomically explained by Kaplan's fibers, which connect the iliotibial tract to the patella. ${ }^{7}$

There is also some evidence in the literature that frontal plane knee alignment is not the only kinematic abnormality in patients with PFP. White et $\mathrm{al}^{47}$ found significant hamstring tightness in patients relative to the control group. Patil et $\mathrm{al}^{48}$ showed by electromyography that the lateral hamstrings of PFP patients contracted earlier than their medial hamstrings during isometric exercises. Besier et $\mathrm{al}^{49}$ demonstrated that patients with PFPS have greater cocontraction of the quadriceps and hamstrings than do controls without symptoms of PFPS. By this mechanism, PFP patients might experience greater joint contact forces and joint stresses than healthy subjects. In addition, Tsuji et al found that elderly patients with PFP have less sacral inclination than healthy controls and referred this condition as "knee-spine syndrome". ${ }^{50}$ In a study by Teng et al, a higher knee flexion moment during the second half of the stance phase of gait was associated with PFOA progression. ${ }^{51}$ However, although the studies reviewed here report several muscular imbalances that have been observed in patients with PFP, altered hip muscle function is considered to be the main cause of dynamic valgus malalignment in PFP patients. ${ }^{7}$

\section{Foot disorders}

Hip muscle dysfunction is likely not the only factor contributing to dynamic valgus. There is strong evidence that, in some patients, foot disorders or deformities such as increased rearfoot eversion or pes pronatus valgus contribute to functional valgus. ${ }^{52-56}$ This deformity can cause internal rotation of the tibia in patients with PFPS. ${ }^{34}$

A systematic review described the kinematic gait characteristics of patients with PFPS. ${ }^{54}$ These gait characteristics included increased rearfoot eversion at heel strike, delayed timing of peak rearfoot eversion, and reduced rearfoot eversion range relative to controls. ${ }^{52}$ Other studies have examined midfoot and forefoot abnormalities in patient with PFP. ${ }^{53,55}$ Mølsgaard et al also found increased navicular drop, navicular drift, and dorsiflexion in high-school students with PFP relative to unaffected students. ${ }^{54}$ Barton et $\mathrm{al}^{53}$ demonstrated that PFP patients had greater forefoot abduction than a healthy control group. 
In conclusion, the literature provides evidence that in a subgroup of PFP patients, rearfoot and forefoot abnormalities contribute to the pathogenesis of dynamic valgus.

\section{Psychological factors}

Several studies have shown that pain and knee function can be associated with psychological factors in some patients with PFP ${ }^{57-61}$ For example, Thomeé et a ${ }^{61}$ found that the pain experience and pain coping in PFP patients were similar to those in other groups of patients with chronic pain. However, patients suffering from PFP had higher scores on the pain catastrophizing scale compared with other chronic pain patients. Similarly, Jensen et $\mathrm{al}^{57}$ reported that patients with PFPS have greater levels of mental distress and worse self-perceived health than healthy subjects. Two studies have identified fear-avoidance beliefs about physical activity as a psychological predictor of pain and dysfunction in PFP patients. ${ }^{60}$

In conclusion, there is evidence that psychological factors could modify the severity of PFP.

\section{Neurophysiological sources of pain in patients with PFP}

The exact source of the pain experienced by patients with PFP is unclear. Most likely the pain develops in the insertions of the extensor muscles or within the subchondral bone. ${ }^{7}$ In an experimental arthroscopy performed using local skin anesthesia, a patient experienced strong pain when the probe touched their retinacula, Hoffas fat pad, or peripatellar synovium. ${ }^{62}$ Several neurotransmitters, including substance $\mathrm{P}$, neurofilament protein, S-100 protein, and neural growth factor, have been found within these structures. ${ }^{63,64}$ These findings suggest that retinacular innervation may play a key role in the development of anterior knee pain. However, there is also evidence that the pain may also originate in the subchondral bone; a PET CT study demonstrated increased metabolic bone activity in patients with PFP. ${ }^{65}$

\section{Therapy}

\section{Surgical treatment options for PFP}

Nonsurgical therapy is the treatment of choice for PFPS in young patients. ${ }^{7}$ Kettunen et al ${ }^{64}$ performed a prospective, randomized trial to compare the outcome of arthroscopy combined with a home exercise program versus a home exercise program alone in patients with PFPS. Patients included in their study were between 18 and 40 years old and had persistent ( $>6$ months) symptoms of PFP during jumping, running, squatting, or while going up or downstairs. ${ }^{64}$ Exclusion criteria were OA, patellar instability, osteochondritis dissecans, and jumper's knee. ${ }^{64}$ The following procedures were performed arthroscopically: resection of the medial plicae, debridement of chondral lesions, partial synovectomy, and lateral release. ${ }^{64}$ Fifty-six patients were included in the study and were randomized either to the arthroscopy plus exercise or to the exerciseonly group. ${ }^{64}$ The mean postintervention improvements in the Kujala knee score and VAS scale score for ascending and descending stairs were greater in the arthroscopy group than in the exercise-only group; ${ }^{64}$ however, these differences were not statistically significant. ${ }^{64}$ The health care costs for patients in the arthroscopy group were significantly higher than the costs for patients in the control group. The results of this study must be considered with caution because only 56 patients were included and thus the study might be underpowered. ${ }^{64}$ With a larger patient population, the observed differences in the clinical scores probably would have been significant. ${ }^{64}$ More criticism of this study includes the heterogeneity of the arthroscopic procedures that were actually performed (11 patients had no arthroscopic treatment, 11 patients underwent cartilage shaving, 5 patients underwent plica resection, 1 lateral release, and 2 partial meniscectomies). Only one patient had a grade IV cartilage defect, and all grade III cartilage defects were treated with debridement; thus, the results of this study are not valid for patients with severe cartilage defects. Severe cartilage defects may indicate that cartilage resurfacing procedures such as matrix-augmented chondrogenesis (AMIC) or autologous chondrocyte implantation (ACI) are required. The results of this prospective, randomized trial suggest that arthroscopy might not be the best first-line treatment for patients with PFPS. However, it is likely that a subgroup of patients exists who would benefit from arthroscopic surgery. This subgroup could include patients with structural joint damage such as grade III or IV cartilage defects or with lateral subluxation of the patella. In a previous study, Kettunen et a ${ }^{65}$ showed that among patients with PFPS, those with severe lesions of the patellar cartilage or femoral trochlea subjectively reported more symptoms and functional limitations at followup than those with no lesions or with small cartilage lesions of the patella or femoral trochlea.

In patients with focal III and IV degrees chondral defects, two studies have reported good clinical results after ACI. ${ }^{66,67}$ In both of these studies, a tibial tubercle transfer and lateral release was used to treat patients with patellofemoral malalignment. ${ }^{66,67}$ In a long-term follow-up study, ACI in combination with anteromedialization of the tibial tuberosity resulted in significant amelioration of symptoms and improvement in function with a low incidence of adverse events in patients with isolated, symptomatic, patellar 
chondral defects after a mean follow-up period of more than 7 years. ${ }^{68}$ When cartilage damage is associated with patellar instability, the PFJ should be stabilized by either MPFL reconstruction, tibial tuberosity transfer, or rotational osteotomy. ${ }^{69}$

\section{Surgical treatment options for PFOA}

There is consensus that PFOA should be treated primarily non-operatively. ${ }^{2,70}$ Non-surgical treatment options for PFOA include exercise, knee realignment braces, patellofemoral taping, foot orthoses, and intra-articular injections of hyaluronic acid or platelet-rich plasma.,70-72 Surgical treatment is indicated if adequate pain relief cannot be achieved with non-surgical management. Surgical options for PFOA are anteromedial tibial tuberosity transfer or patellofemoral resurfacing. ${ }^{73}$

\section{Nonsurgical treatment options for PFP}

Two recently published articles describe the guidelines for conservative management of PFP: These publications, the "2016 Patellofemoral Pain Consensus Statement" and the "Best Practice Guidelines", 2,74 both recommended interventions that include exercise, taping, bracing, foot orthoses, and combined therapies. ${ }^{2,74}$ In addition, the "Best Practice Guidelines" recommend acupuncture and patient education. In both publications, these interventions are recommended for young patients with PFPS and for patients with PFOA. ${ }^{2}$ However, PFP treatment should be individualized for each patient; not all patients will require all treatments but scientific evidence to guide the tailoring of interventions is very limited. ${ }^{2,74}$

\section{Exercise}

Most authors agree that exercise therapy is the treatment of choice for patients with PFP. ${ }^{2,74}$ Exercise has been the most well-studied form of PFPS therapy and should aim to correct the dynamic valgus malalignment that occurs in many patients. ${ }^{7}$ Five recently published systematic reviews concluded that exercise had a positive effect on pain and function in patients with PFP. ${ }^{75-79}$ The most effective intervention programs included exercises targeting the hip external rotator and abductor muscles and knee extensor muscles. However, positive results have also been described for exercises that target only muscles acting on the knee and hip. ${ }^{75-79}$ One study showed a positive effect of trunk stabilization exercises, which targeted core muscles such as the rectus abdominis. The results of previous studies suggest that, if knee and hip exercises are recommended, then the patient should begin with the more proximal exercises because they have greater benefit and may cause fewer adverse effects., ${ }^{2,74}$

A positive clinical effect has been described for active stretching exercises, ergometer exercise, squats, static quadriceps exercises, leg presses, active leg raises and lowers, and climbing exercises. ${ }^{7,75-79}$ Open- and closed-chain, balance, and neuromuscular training programs have been described. ${ }^{7,75-79}$ The most frequent duration of the exercise programs was 6 weeks, and the exercises were generally conducted two to four times daily with 10 repetitions per exercise session. ${ }^{775-79}$ Both supervised and home-based exercise programs have been described. ${ }^{7,75-79}$ In several studies, additional interventions such as restriction of symptom-inducing activities, tape, braces, and nonsteroidal anti-inflammatory drugs (NSAIDs) were allowed. ${ }^{7}$

In summary, there is strong evidence that a targeted exercise program is an effective treatment for PFP. ${ }^{7}$ Exercises to treat PFP should target the muscles of the hip, knee (quadriceps and hamstrings), trunk, and the iliotibial tract (Figure 5).

\section{Pharmacological therapy}

The "Best Practice Guidelines" recommend that immediate pain relief should be a priority to gain patient trust. ${ }^{74} \mathrm{~A}$

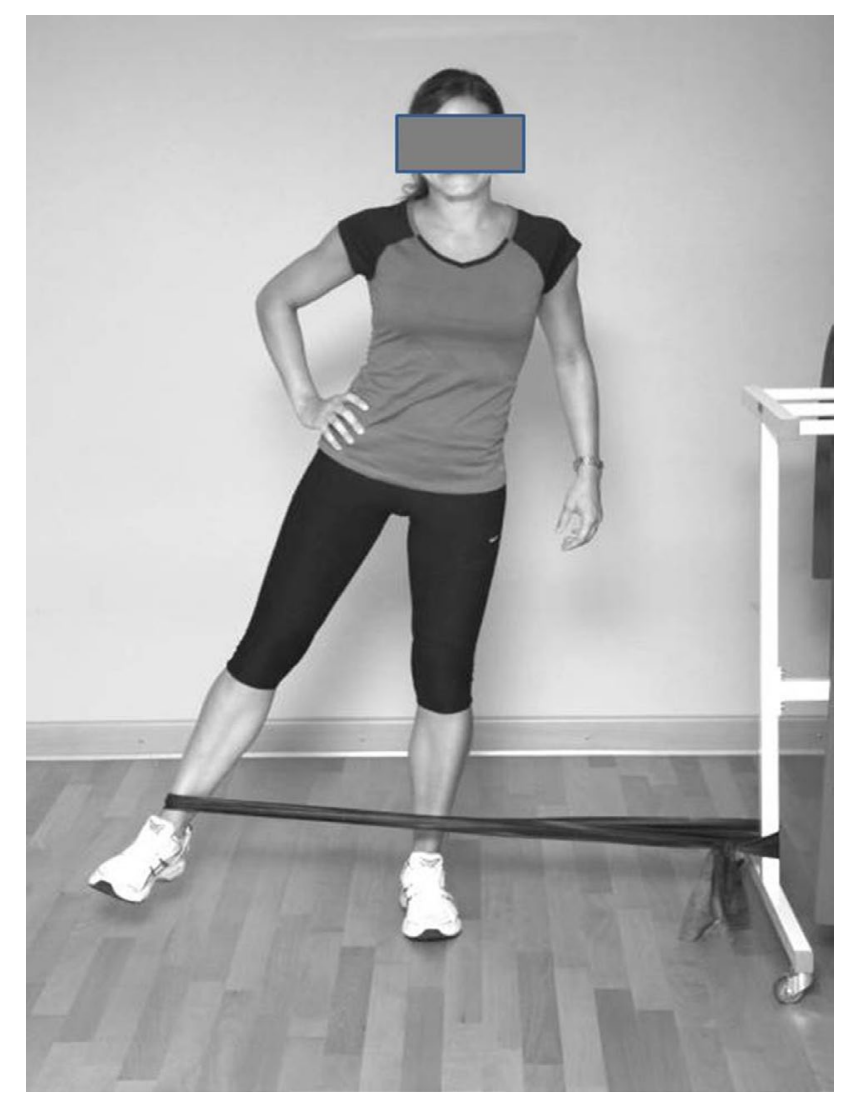

Figure 5 Exercise for hip abductors. 
Cochrane review from 2004 concluded that there was limited evidence that NSAIDs effectively reduced acute anterior knee pain in PFPS patients. ${ }^{7,80}$ More recent systematic reviews of pharmacological treatments for PFP are lacking. The OARSI guideline for the nonoperative treatment of knee OA recommend the use of selective or nonselective NSAIDs for patients without comorbidities. ${ }^{70}$ Thus, despite the limited evidence supporting their use, NSAIDs may be appropriate in patients with PFOA.

\section{Correction of patellar maltracking by taping}

Taping is a popular treatment for athletes with PFPS, ${ }^{81-83}$ in part because the tape strips do not hinder sporting activity. Classical taping technique is different from Kinesio taping. ${ }^{84}$

The most popular classical taping technique for PFPS treatment was described by McConnell $^{85}$ (Figure 6A). The aim of McConnell taping is to correct lateral patellar maltracking and patellar tilt by applying adhesive strips to the skin. ${ }^{85}$ This technique has been recommended for athletes with PFPS for use during training and competition. ${ }^{81}$

A recent systematic review concluded that there is moderate evidence that customized classical taping provides pain reduction that is superior to that provided by untailored tape application, and there is limited evidence that tailored taping provides better pain relief than exercise alone after 4 weeks of each intervention. ${ }^{83}$ There was also evidence that tailored taping stimulated earlier $\mathrm{m}$. vastus medialis activation relative to $\mathrm{m}$. vastus lateralis activation. ${ }^{83}$ These findings suggest that in addition to its biomechanical effects, taping may also have neuromotor effects. ${ }^{83}$ However, the finding that even sham tape application produces a clinically meaningful reduction in knee pain in patients with PFPS suggests that placebo or sensory skin effects may contribute to the benefits of taping. ${ }^{86}$ It should be noted that the effects of tape on pain reduction

A

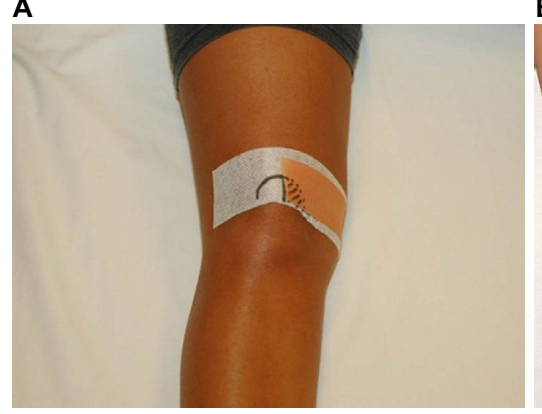

Figure 6 Tape applications for patients with PFP.

Notes: (A) Application of a classical McConnell tape. (B) Application of a Kinesio tape. have only been investigated in short-term studies (12-week follow-up). ${ }^{7,83}$ The long-term effects of tape on anterior knee pain have not been established. Therefore, current evidence supports the use of tape for temporary pain relief in the treatment of anterior knee pain in PFPS patients, ${ }^{83}$ but the benefits of long-term taping have not been established. According to the "2016 Patellofemoral Pain Consensus Statement" and the "Best Practice Guidelines", tailored classical tape application can be recommended in combination with other treatments, such as exercise. . $^{2,74}$

During the past few years, the use of Kinesio tape has become increasingly popular (Figure 6B). Kinesio taping uses a different approach than classical taping; in Kinesio taping, the elastic tape strips are used to increase the space between muscle and fascia. In this way, Kinesio taping can stimulate cutaneous mechanoreceptors and improve knee proprioception. ${ }^{81}$ The tape can either stimulate muscle activity or prevent muscle overuse, depending on how it is applied. ${ }^{87}$

A recently published systematic review compared studies that used Kinesio taping with studies that used the classic McConnell taping technique. ${ }^{81}$ This review reported that both taping techniques had a positive effect on pain and quality of life, while meta-analysis showed that Kinesio taping provided more effective pain relief than McConnell taping. ${ }^{81}$ However, both methods work in different ways. Kinesio taping increases muscle flexibility, improves motor function, and has a moderate effect on muscle activity in PFPS patients compared with controls. ${ }^{81}$ Meta-analysis has shown a small effect on pain reduction and motor function improvement and a moderate effect on muscle activity, in PFPS patients treated with Kinesio taping. ${ }^{81}$ The positive effects of McConnell taping have been attributed to correction of patellar maltracking. ${ }^{81}$ The authors of this systematic review concluded that both patellar taping techniques can be useful in the treatment of PFPS patients. ${ }^{81}$

\section{Patellar braces}

A disadvantage of taping is that its effects decrease over time. Patellar braces are an alternative option; these apply an external, medially directed force that may counteract lateral patella maltracking (Figure 7 ). ${ }^{7}$ Biomechanical studies have shown that patellar braces can alter patellar tracking. Draper et $\mathrm{al}^{88}$ demonstrated using real-time MRI that a knee brace could reduce patellar lateralization and tilt in women with PFPS. Becher et al $^{89}$ demonstrated that a dynamic realignment brace significantly affected the reduction in the three patellar height ratios, patellar tilt angle, and bisect offset, as well as TT-TG distance. This dynamic realignment brace 


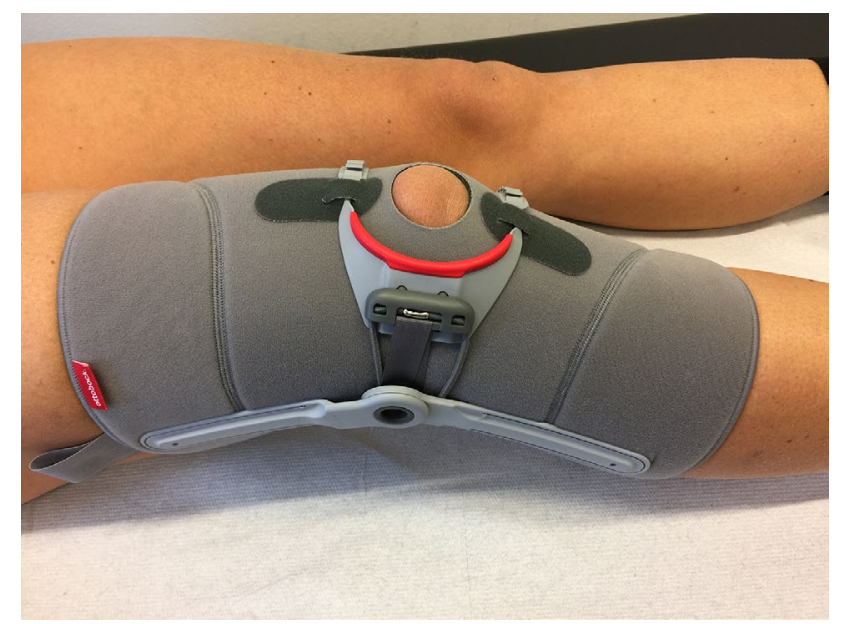

Figure 7 Example of a patella realignment brace (Agillium Patella pro; Otto Bock, Duderstdt, Germany).

(Agilium Patella pro; Otto Bock, Duderstadt, Germany) applies a medially directed force only when the knee is flexed to between 0 and $30^{\circ}$, which is when the patella is not stabilized by the trochlea. Callaghan et $\mathrm{al}^{90}$ have shown that use of a patellar brace alters position and increases contact area between the patella and the femoral trochlea in patients with patellofemoral OA.

A 2015 meta-analysis found insufficient evidence to recommend the use of knee braces in the treatment of patients with PFPS. ${ }^{91}$ However, two recently published, prospective, randomized studies did demonstrate a positive effect of knee braces in patients with PFPS and PFOA. Callaghan et al ${ }^{92}$ demonstrated that a patellar brace (Bioskin Patellar Tracking Q Brace; Ossur UK, Manchester, England) relieved knee pain in patients with PFOA. This was attributed to a reduction in the volume of bone marrow lesions in the targeted compartment of the knee. Petersen et $\mathrm{al}^{93}$ examined the effect of a dynamic realignment brace (Patella pro) in patients with PFPS. After 6 and 12 weeks of treatment, they found that use of the brace led to better outcomes in patients with PFPS than exercise alone. ${ }^{93}$ However, 1 year later, this positive effect had diminished. ${ }^{93}$ The results of both of these randomized trials suggest that the use of realignment braces in patients with PFP is justified.

\section{Foot orthotics}

Foot abnormalities such as increased rearfoot eversion and pes pronatus can favor internal rotation of the tibia and create a functional malalignment of the leg. ${ }^{7}$ Therefore, insoles or foot orthotics may be a treatment option for patients with PFP. $^{7}$ A systematic review published in 2010 found limited evidence that prefabricated foot orthoses reduce the range of transverse plane knee rotation, which has been associated with short-term improvements in individuals with PFP. ${ }^{94} \mathrm{~A}$ recently published systematic review of 11 clinical studies demonstrated that the use of foot orthotics had significant effects on pain and function in patients with PFP. ${ }^{95}$ This review also identified a slight effect of foot orthoses on lower limb kinematics and muscle activation. ${ }^{95}$ However, other studies have not found any effects of foot orthoses on clinical outcome in patients with PFP. ${ }^{96}$ This heterogeneity in study results demonstrates that there may be substantial variations in individual responses to this treatment and that foot orthoses may not be beneficial for all patients with PFP. ${ }^{2}$ Patients with foot abnormalities, such as those with increased rearfoot eversion or pes pronatus, may benefit the most from foot orthotics. Therefore, foot orthoses might be a treatment option for patients with the combination of disorders of foot posture and PFPS. ${ }^{7}$

\section{Conclusion}

The existing evidence regarding the pathogenesis of PFP can be summarized in an algorithm shown in Figure 8. Patellar maltracking owing to a functional malalignment or dynamic valgus may be an underlying cause of PFP. Possible causes of functional valgus may be decreased hip muscle strength or foot abnormalities. Secondary consequences include quadriceps imbalance, hamstring tightness, or iliotibial tract tightness (Figure 8). The clinical significance of these findings is that an individually tailored, multimodal treatment plan for each patient based on their underlying pathology is necessary. Options for this treatment plan include exercise programs that target the muscles of the lower extremity, hip, and trunk, patellar taping or bracing, foot orthoses, and the short-term use of NSAIDs (Figure 9). The greatest evidence exists for exercises. If the clinical examination shows signs of hip muscle weakness, these muscles should be addressed. Foot orthoses might be a treatment option for patients with the combination of disorders of foot posture and PFPS. Bracing and taping can support patella tracking. NSAIDs should be used for acute episode of pain.

\section{Disclosure}

Wolf Petersen, Ingo Rembitzki, and Christian Liebau receive consultant fees from Otto Bock Health Care (Duderstadt, Germany). The authors report no other conflicts of interest in this work. 


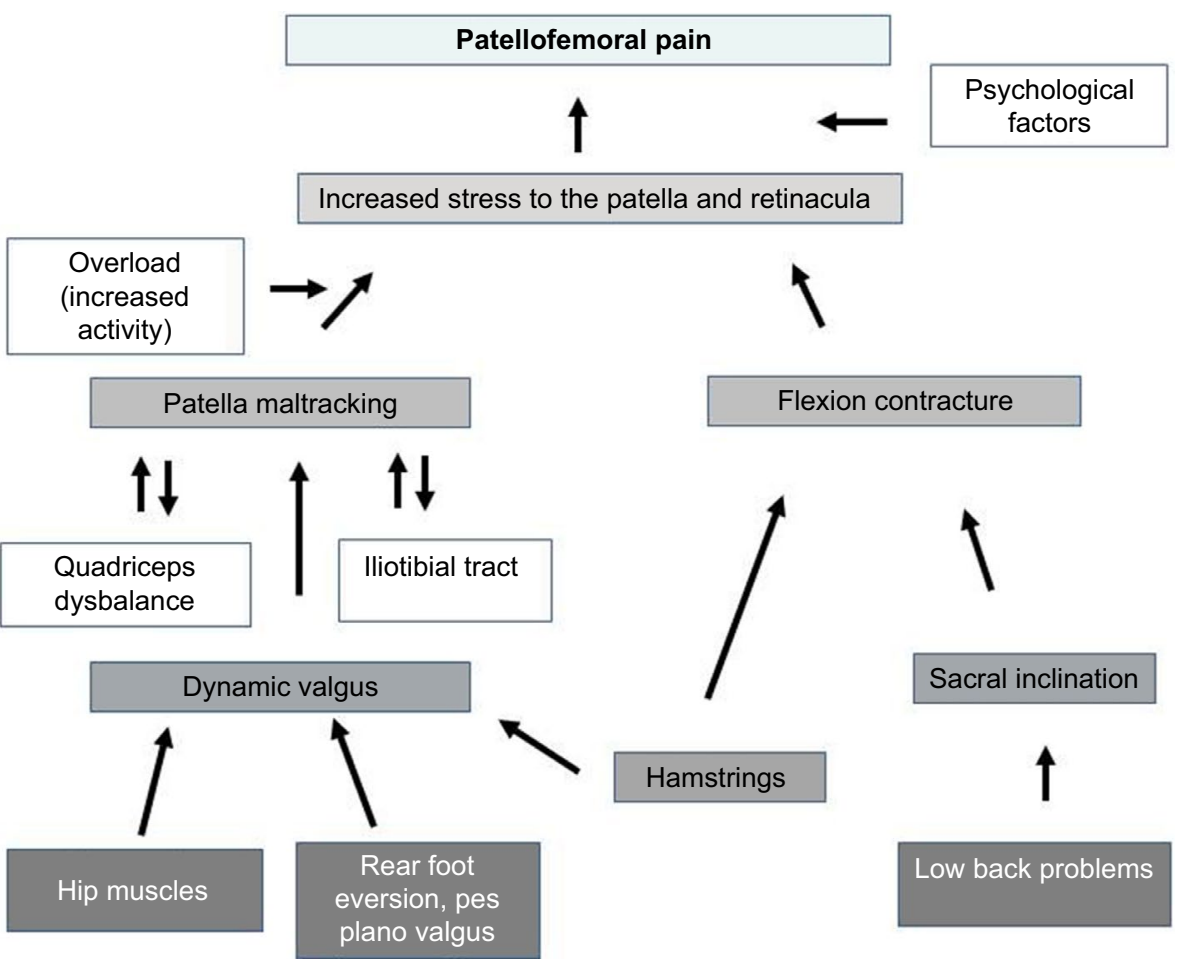

Figure 8 Algorithm for the pathogenesis of PFPS.

Notes: Patellar maltracking due to a functional malalignment in the coronal and sagittal planes may be an underlying cause of patellofemoral pain. Possible causes of functional malalignment may be decreased hip muscle strength, low back problems, or foot abnormalities. Secondary consequences include quadriceps imbalance, hamstring tightness, or iliotibial tract tightness. Psychological factors could additionally modify the pain sensation.

Abbreviation: PFPS, patellofemoral pain syndrome.
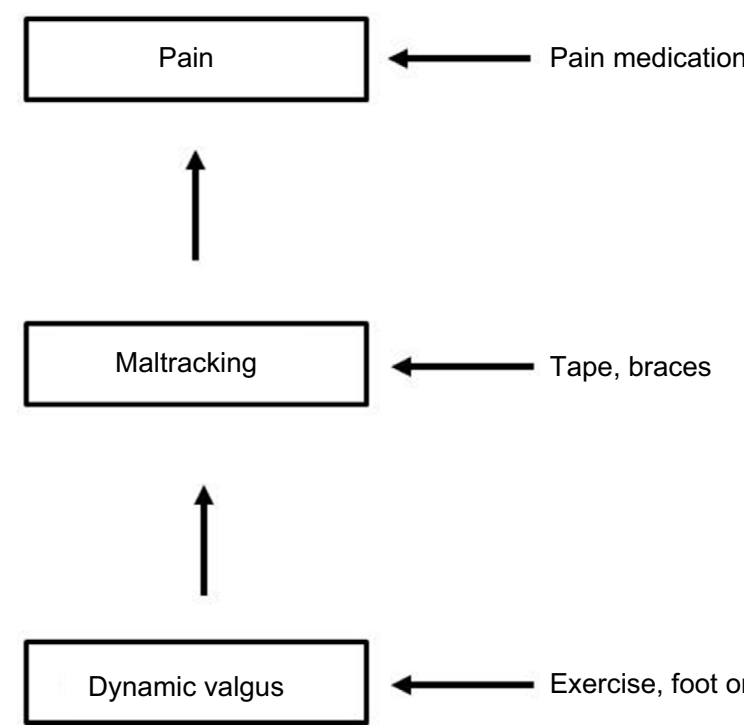

Figure 9 Algorithm for the treatment of PFPS.

Abbreviation: PFPS, patellofemoral pain syndrome.

\section{References}

1. Crossley KM, Stefanik JJ, Selfe J, et al. Patellofemoral pain consensus statement from the 4th International Patellofemoral Pain Research Retreat, Manchester. Part 1: terminology, definitions, clinical examination, natural history, patellofemoral osteoarthritis and patient-reported outcome measures. Br J Sports Med. 2016;50(14): 839-843.
2. Crossley KM, van Middelkoop M, Callaghan MJ, et al. Patellofemoral pain consensus statement from the 4th International Patellofemoral Pain Research Retreat, Manchester. Part 2: recommended physical interventions (exercise, taping, bracing, foot orthoses and combined interventions). Br J Sports Med. 2016;50(14):844-852.

3. Clement DB, Tauton JE, Smart GW, McNicol KL. A survey of overuse running injuries. Phys Sportsmed. 1981;9(1):47-58. 
4. Dierks TA, Manal KT, Hamill J, Davis I. Lower extremity kinematics in runners with patellofemoral pain during a prolonged run. Med Sci Sports Exerc. 2011;43(4):693-700.

5. Rathleff MS, Rasmussen S, Olesen JL. Unsatisfactory long-term prognosis of conservative treatment of patellofemoral pain syndrome. Ugeskr Laeger. 2012;174(15):1008-1013.

6. Foss KD, Myer GD, Magnussen RA, Hewett TE. Diagnostic differences for anterior knee pain between sexes in adolescent basketball players. $J$ Athl Enhanc. 2014;3(1):1814-1820.

7. Petersen W, Ellermann A, Gösele-Koppenburg A, et al. Patellofemoral pain syndrome. Knee Surg Sports Traumatol Arthrosc. 2014;22(10): 2264-2274.

8. Thomas MJ, Wood L, Selfe J, Peat G. Anterior knee pain in younger adults as a precursor to subsequent patellofemoral osteoarthritis: a systematic review. BMC Musculoskelet Disord. 2010;11:201-210.

9. van der Heijden RA, de Kanter JL, Bierma-Zeinstra SM, et al. Structural abnormalities on magnetic resonance imaging in patients with patellofemoral pain: a cross-sectional case-control study. Am J Sports Med. 2016;44(9):2339-2346.

10. Drew BT, Redmond AC, Smith TO, Penny F, Conaghan PG. Which patellofemoral joint imaging features are associated with patellofemoral pain? Systematic review and meta-analysis. Osteoarthritis Cartilage. 2016;24(2):224-236.

11. Wilson NA, Press JM, Koh JL, Hendrix RW, Zhang LQ. In vivo noninvasive evaluation of abnormal patellar tracking during squatting in patients with patellofemoral pain. J Bone Joint Surg Am. 2009; 91(3):558-566.

12. Hunter DJ, Zhang YQ, Niu JB, et al. Patella malalignment, pain and patellofemoral progression: the Health ABC Study. Osteoarthritis Cartilage. 2007;15(10):1120-1127.

13. Stefanik JJ, Gross KD, Guermazi A, et al. The relation of MRI-detected structural damage in the medial and lateral patellofemoral joint to knee pain: the Multicenter and Framingham Osteoarthritis Studies. Osteoarthritis Cartilage. 2015;23(4):565-570.

14. Sheehan FT, Derasari A, Fine KM, Brindle TJ, Alter KE. Q-angle and J-sign: indicative of maltracking subgroups in patellofemoral pain. Clin Orthop Relat Res. 2010;468(1):266-275.

15. Macri EM, Stefanik JJ, Khan KM, et al. Is tibiofemoral or patellofemoral alignment or trochlear morphology associated with patellofemoral osteoarthritis? A systematic review. Arthritis Care Res (Hoboken). 2016;68(10):1453-1470.

16. Horton MG, Hall TL. Quadriceps femoris muscle angle:normal values and relationships with gender and selected skeletal measures. Phys Ther. 1989;69(11):17-21.

17. Lankhorst NE, Bierma-Zeinstra SM, van Middelkoop M. Factors associated with patellofemoral pain syndrome: a systematic review. Br J Sports Med. 2013;47(4):193-206.

18. Park SK, Stefanyshyn DJ. Greater Q angle may not be a risk factor of patellofemoral pain syndrome. Clin Biomech (Bristol, Avon). 2011;26(4):392-396.

19. Almeida GP, Silva AP, França FJ, Magalhães MO, Burke TN, Marques AP. mQ-angle in patellofemoral pain: relationship with dynamic knee valgus, hip abductor torque, pain and function. Rev Bras Orthop. 2016;51(2):181-186.

20. Kwon O, Yun M, Lee W. Correlation between intrinsic patellofemoral pain syndrome in young adults and lower extremity biomechanics. J Phys Ther Sci. 2014;26(7):961-964.

21. Pappas E, Wong-Tom WM. Prospective predictors of patellofemoral pain syndrome: a systematic review with meta-analysis. Sports Health. 2012;4(2):115-120.

22. Dierks TA, Manal KT, Hamill J, Davis I. Lower extremity kinematics in runners with patellofemoral pain during a prolonged run. Med Sci Sports Exerc. 2011;43(4):693-700.

23. Myer GD, Ford KR, Barber Foss KD, et al. The incidence and potential pathomechanics of patellofemoral pain in female athletes. Clin Biomech (Bristol, Avon). 2010;25(7):700-707.
24. Nakagawa TH, Moriya ET, Maciel CD, Serrão FV. Trunk, pelvis, hip, and knee kinematics, hip strength, and gluteal muscle activation during a single-leg squat in males and females with and without patellofemoral pain syndrome. J Orthop Sports Phys Ther. 2012;42(6):491-501.

25. Souza RB, Powers CM. Differences in hip kinematics, muscle strength, and muscle activation between subjects with and without patellofemoral pain. J Orthop Sports Phys Ther. 2009;39(1):12-19.

26. Ford KR, Myer GD, Hewett TE. Valgus knee motion during landing in high school female and male basketball players. Med Sci Sports Exerc. 2003;35(10):1745-1750.

27. Ford KR, Myer GD, Toms HE, Hewett TE. Gender differences in the kinematics of unanticipated cutting in young athletes. Med Sci Sports Exerc. 2005;37(1):124-129.

28. Fulkerson JP. The etiology of patellofemoral pain in young, active patients: a prospective study. Clin Orthop Relat Res. 1983;179: 129-133.

29. Souza RB, Draper CE, Fredericson M, Powers CM. Femur rotation and patellofemoral joint kinematics: a weight-bearing magnetic resonance imaging analysis. J Orthop Sports Phys Ther. 2010;40(5):2772-2785.

30. Crossley KM, Zhang WJ, Schache AG, Bryant A, Cowan SM. Performance on the single-leg squat task indicates hip abductor muscle function. Am J Sports Med. 2011;39(4):866-873.

31. Cavazzuti L, Merlo A, Orlandi F, Campanini I. Delayed onset of electromyographic activity of vastus medialis obliquus relative to vastus lateralis in subjects with patellofemoral pain syndrome. Gait Posture. 2010;32(3):290-295.

32. Chen HY, Chien CC, Wu SK, Liau JJ, Jan MH. Electromechanical delay of the vastus medialis obliquus and vastus lateralis in individuals with patellofemoral pain syndrome. J Orthop Sports Phys Ther. 2012;42(9):791-796.

33. Cowan SM, Bennell KL, Hodges PW, Crossley KM, McConnell J. Delayed onset of electromyographic activity of vastus medialis obliquus relative to vastus lateralis in subjects with patellofemoral pain syndrome. Arch Phys Med Rehabil. 2001;82(2):183-189.

34. Hart HF, Ackland DC, Pandy MG, Crossley KM. Quadriceps volumes are reduced in people with patellofemoral joint osteoarthritis. Osteoarthritis Cartilage. 2012;20(8):863-868.

35. Peat G, Duncan RC, Wood LRJ, et al. Clinical features of symptomatic patellofemoral joint osteoarthritis. Arthritis Res Ther. 2012;14(2):63-69.

36. Farrokhi S, Piva SR, Gil AB, Oddis CV, Brooks MM, Fitzgerald GK. Association of severity of coexisting patellofemoral disease with increased impairments and functional limitations in patients with knee osteoarthritis. Arthritis Care Res (Hoboken). 2013;65(4):544-551.

37. Fok LA, Schache AG, Crossley KM, Lin YC, Pandy MG. Patellofemoral joint loading during stair ambulation in people with patellofemoral osteoarthritis. Arthritis Rheum. 2013;65(8):2059-2069.

38. Amin S, Baker K, Niu J, et al. Quadriceps strength and the risk of cartilage loss and symptom progression in knee osteoarthritis. Arthritis Rheum. 2009;60(1):189-198.

39. Baldon Rde M, Nakagawa TH, Muniz TB, Amorim CF, Maciel CD, Serrão FV. Eccentric hip muscle function in females with and without patellofemoral pain syndrome. J Athl Train. 2009;44(5):490-496.

40. Bolgla LA, Malone TR, Umberger BR, Uhl TL. Hip strength and hip and knee kinematics during stair descent in females with and without patellofemoral pain syndrome. J Orthop Sports Phys Ther. 2008;38(1):12-16.

41. Brent JL, Myer GD, Ford KR, Hewett TE. A longitudinal examination of hip abduction strength in adolescent males and females. Med Sci Sports Exerc. 2008;39:34-45.

42. Prins MR, van der Wurff P. Females with patellofemoral pain syndrome have weak hip muscles: a systematic review. Aust J Physiother. 2009;55(1):9-15.

43. Padua DA, Marshall SW, Beutler AI, et al. Predictors of knee valgus angle during a jump-landing task. Med Sci Sports Exerc. 2005; $37: 398-404$ 
44. Crossley KM, Dorn TW, Ozturk H, van den Noort J, Schache AG, Pandy MG. Altered hip muscle forces during gait in people with patellofemoral osteoarthritis. Osteoarthritis Cartilage. 2012;20(11):1243-1249.

45. Pohl MB, Patel C, Wiley JP, Ferber R. Gait biomechanics and hip muscular strength in patients with patellofemoral osteoarthritis. Gait Posture. 2013;37(3):440-444.

46. Wu CC, Shih $\mathrm{CH}$. The influence of iliotibial tract on patellar tracking. Orthopedics. 2004;27(2):199-203.

47. White LC, Dolphin P, Dixon J. Hamstring length in patellofemoral pain syndrome. Physiotherapy. 2009;95(1):24-28.

48. Patil S, Dixon J, White LC, Jones AP, Hui AC. An electromyographic exploratory study comparing the difference in the onset of hamstring and quadriceps contraction in patients with anterior knee pain. Knee. 2011;18(5):329-332.

49. Besier TF, Fredericson M, Gold GE, Beaupré GS, Delp SL. Knee muscle forces during walking and running in patellofemoral pain patients and pain-free controls. J Biomech. 2009;42(7):898-905.

50. Barton CJ, Levinger P, Menz HB, Webster KE. Kinematic gait characteristics associated with patellofemoral pain syndrome: a systematic review. Gait Posture. 2009;30(4):405-416.

51. Barton CJ, Levinger P, Webster KE, Menz HB. Walking kinematics in individuals with patellofemoral pain syndrome: a case-control study. Gait Posture. 2011;33(2):286-289.

52. Barton CJ, Levinger P, Crossley KM, Webster KE, Menz HB. The relationship between rearfoot, tibial and hip kinematics in individuals with patellofemoral pain syndrome. Clin Biomech (Bristol, Avon). 2012;27(7):702-705.

53. Barton CJ, Bonanno D, Levinger P, Menz HB. Foot and ankle characteristics in patellofemoral pain syndrome: a case control and reliability study. J Orthop Sports Phys Ther. 2010;40(5):286-296.

54. Mølgaard M, Rathleff MS, Simonsen O. Patellofemoral pain syndrome and its association with hip, ankle, and foot function in 16- to 18-year-old high school students: a single-blind case-control study. J Am Podiatr Med Assoc. 2011;101(3):215-222.

55. Jensen R, Hystad T, Baerheim A. Knee function and pain related to psychological variables in patients with long-term patellofemoral pain syndrome. J Orthop Sports Phys Ther. 2005;35(9):594-600.

56. Jensen R, Hystad T, Kvale A, Baerheim A. Quantitative sensory testing of patients with long lasting patellofemoral pain syndrome. Eur J Pain. 2007;11(6):665-676.

57. Piva SR, Fitzgerald GK, Wisniewski S, Delitto A. Predictors of pain and function outcome after rehabilitation in patients with patellofemoral pain syndrome. J Rehabil Med. 2009;41(8):604-612.

58. Piva SR, Fitzgerald GK, Irrgang JJ, et al. Associates of physical function and pain in patients with patellofemoral pain syndrome. Arch Phys Med Rehabil. 2009;90(2):285-295.

59. Thomeé $\mathrm{P}$, Thomeé R, Karlsson J. Patellofemoral pain syndrome: pain, coping strategies and degree of well-being. Scand J Med Sci Sports 2002;12(5):276-281.

60. Dye SF. The pathophysiology of patellofemoral pain: a tissue homeostasis perspective. Clin Orthop Relat Res. 2005;436:100-110.

61. Sanchis-Alfonso V, Roselló-Sastre E. Immunohistochemical analysis for neural markers of the lateral retinaculum in patients with isolated symptomatic patellofemoral malalignment. A neuroanatomic basis for anterior knee pain in the active young patient. Am J Sports Med. 2000;28(5):725-731.

62. Wojtys EM, Beaman DN, Glover RA, Janda D. Innervation of the human knee joint by substance-P fibers. Arthroscopy. 1990;6(4) 254-263.

63. Draper CE, Fredericson M, Gold GE, et al. Patients with patellofemoral pain exhibit elevated bone metabolic activity at the patellofemoral joint. J Orthop Res. 2012;30(2):209-213.

64. Kettunen JA, Harilainen A, Sandelin J, et al. Knee arthroscopy and exercise versus exercise only for chronic patellofemoral pain syndrome: a randomized controlled trial. BMC Med. 2007;5:38-45.
65. Kettunen JA, Visuri T, Harilainen A, Sandelin J, Kujala UM. Primary cartilage lesions and outcome among subjects with patellofemoral pain syndrome. Knee Surg Sports Traumatol Arthrosc. 2005;13(2):31-34.

66. Ebert JR, Fallon M, Smith A, Janes GC, Wood DJ. Prospective clinical and radiologic evaluation of patellofemoral matrix-induced autologous chondrocyte implantation. Am J Sports Med. 2015;43(6):1362-1372.

67. Meyerkort D, Ebert JR, Ackland TR, et al. Matrix-induced autologous chondrocyte implantation (MACI) for chondral defects in the patellofemoral joint. Knee Surg Sports Traumatol Arthrosc. 2014;22(10):2522-2523.

68. Gillogly SD, Arnold RM. Autologous chondrocyte implantation and anteromedialization for isolated patellar articular cartilage lesions: 5- to 11-year follow-up. Am J Sports Med. 2014;42(4):912-920.

69. Petersen W, Forkel P, Achtnich A. Chronic patellofemoral instability. Unfallchirurg. 2012;115(5):397-409.

70. McAlindon TE, Bannuru RR, Sullivan MC, et al. OARSI guidelines for the non-surgical management of knee osteoarthritis. Osteoarthritis Cartilage. 2014;22(3):363-388.

71. Campbell KA, Erickson BJ, Saltzman BM, et al. Is local viscosupplementation injection clinically superior to other therapies in the treatment of osteoarthritis of the knee: a systematic review of overlapping meta-analyses. Arthroscopy. 2015;31(10):2036-2045.

72. Meheux CJ, McCulloch PC, Lintner DM, Varner KE, Harris JD. Efficacy of intra-articular platelet-rich plasma injections in knee osteoarthritis: a systematic review. Arthroscopy. 2016;32(3):495-505.

73. Mosier BA, Arendt EA, Dahm DL, Dejour D, Gomoll AH. Management of patellofemoral arthritis: from cartilage restoration to arthroplasty. J Am Acad Orthop Surg. 2016;24(11):163-173.

74. Barton CJ, Lack S, Hemmings S, Tufail S, Morrissey D. The 'Best Practice Guide to Conservative Management of Patellofemoral Pain': incorporating level 1 evidence with expert clinical reasoning. $\mathrm{Br} J$ Sports Med. 2015;49(14):923-923.

75. Harvie D, O'Leary T, Kumar S. A systematic review of randomized controlled trials on exercise parameters in the treatment of patellofemoral pain: what works? J Multidiscip Healthc. 2011;4:383-392.

76. Alba-Martín P, Gallego-Izquierdo T, Plaza-Manzano G, RomeroFranco N, Núñez-Nagy S, Pecos-Martín D. Effectiveness of therapeutic physical exercise in the treatment of patellofemoral pain syndrome: a systematic review. J Phys Ther Sci. 2015;27(7):2387-2390.

77. Peters JS, Tyson NL. Proximal exercises are effective in treating patellofemoral pain syndrome: a systematic review. Int J Sports Phys Ther. 2013;8(5):689-700.

78. Thomson C, Krouwel O, Kuisma R, Hebron C. The outcome of hip exercise in patellofemoral pain: a systematic review. Man Ther. 2016;26:1-30.

79. van der Heijden RA, Lankhorst NE, van Linschoten R, Bierma-Zeinstra SM, van Middelkoop M. Exercise for treating patellofemoral pain syndrome. Cochrane Database Syst Rev. 2015;1:CD010387.

80. Heintjes E, Berger MY, Bierma-Zeinstra SM, Bernsen RM, Verhaar JA, Koes BW. Pharmacotherapy for patellofemoral pain syndrome. Cochrane Database Syst Rev. 2004;3:CD003470.

81. Chang WD, Chen FC, Lee CL, Lin HY, Lai PT. Effects of Kinesio Taping versus McConnell Taping for patellofemoral pain syndrome: a systematic review and meta-analysis. Evid Based Complement Alternat Med. 2015;2015:471208.

82. Warden SJ, Hinman RS, Watson MA Jr, Avin KG, Bialocerkowski AE, Crossley KM. Patellar taping and bracing for the treatment of chronic knee pain: a systematic review and meta-analysis. Arthritis Rheum. 2008;59(1):73-78.

83. Barton C, Balachandar V, Lack S, Morrissey D. Patellar taping for patellofemoral pain: a systematic review and meta-analysis to evaluate clinical outcomes and biomechanical mechanisms. Br J Sports Med. 2014;48(6):417-424.

84. Campolo M, Babu J, Dmochowska K, Scariah S, Varughese J. A comparison of two taping techniques (Kinesio and McConnell) and their effect on anterior knee pain during functional activities. Int J Sports Phys Ther. 2013;8(2):105-110. 
85. Callaghan MJ, Selfe J. Patellar taping for patellofemoral pain syndrome in adults. Cochrane Database Syst Rev. 2012;4:CD006717.

86. McConnell J. A novel approach to pain relief pre-therapeutic exercise. J Sci Med Sport. 2000;3(3):325-334.

87. Osorio JA, Vairo GL, Rozea DG, et al. The effects of two therapeutic patellofemoral taping techniques on strength, endurance, and pain responses. Phys Ther Sport. 2013;14(4):199-206.

88. Draper CE, Besier TF, Santos JM, et al. Using real-time MRI to quantify altered joint kinematics in subjects with patellofemoral pain and to evaluate the effects of a patellar brace or sleeve on joint motion. J Orthop Res. 2009;27(5):571-577.

89. Becher C, Schumacher T, Fleischer B, Ettinger M, Smith T, Ostermeier $\mathrm{S}$. The effects of a dynamic patellar realignment brace on disease determinants for patellofemoral instability in the upright weight-bearing condition. J Orthop Surg Res. 2015;10:126.

90. Callaghan MJ, Guney H, Reeves ND, et al. A knee brace alters patella position in patellofemoral osteoarthritis: a study using weight bearing magnetic resonance imaging. Osteoarthritis Cartilage. 2016;24(12): 2055-2060.
91. Smith TO, Drew BT, Meek TH, Clark AB. Knee orthoses for treating patellofemoral pain syndrome. Cochrane Database Syst Rev. 2015;12:CD010513.

92. Callaghan MJ, Parkes MJ, Hutchinson CE, et al. A randomised trial of a brace for patellofemoral osteoarthritis targeting knee pain and bone marrow lesions. Ann Rheum Dis. 2015;74(6):1164-1170.

93. Petersen W, Ellermann A, Rembitzki IV, et al. Evaluating the potential synergistic benefit of a realignment brace on patients receiving exercise therapy for patellofemoral pain syndrome: a randomized clinical trial. Arch Orthop Trauma Surg. 2016;136(7):975-982.

94. Barton CJ, Munteanu SE, Menz HB, Crossley KM. The efficacy of foot orthoses in the treatment of individuals with patellofemoral pain syndrome: a systematic review. Sports Med. 2010;40(5):377-395.

95. Ahlhelm A, Alfuth M. The influence of foot orthoses on patellofemoral pain syndrome: a systematic analysis of the literature. Sportverletz Sportschaden. 2015;29(2):107-117.

96. Hossain M, Alexander P, Burls A, Jobanputra P. Foot orthoses for patellofemoral pain in adults. Cochrane Database Syst Rev. 2011;1:CD008402.
Open Access Journal of Sports Medicine

\section{Publish your work in this journal}

The Open Access Journal of Sports Medicine is an international, peer-reviewed, open access journal publishing original research, reports, reviews and commentaries on all areas of sports medicine. The journal is included on PubMed. The manuscript management system is completely online and includes a very quick and fair
Dovepress

peer-review system. Visit http://www.dovepress.com/testimonials.php to read real quotes from published authors. 